\title{
Symptomatic unruptured capillary telangiectasia of the brain stem: report of three cases and review of the literature
}

\author{
C Scaglione, F Salvi, P Riguzzi, M Vergelli, C A Tassinari, M Mascalchi
}

\begin{abstract}
Three young patients with transient or intermittent focal neurological signs suggesting brain stem involvement are described, in whom high field MRI showed focal areas of hyperintensity in $T 2$ weighted spin echo images, hypointensity in $\mathrm{T} 2 \star$ weighted gradient echo images, and enhancement in postcontrast $T 1$ weighted images consistent with unruptured capillary telangiectasia of the brain stem. The first patient was a 28 year old woman who complained of recurrent left ear tinnitus, exacerbated during the menstrual period; MRI demonstrated that the vascular anomaly involved the left acoustic pathway. The second patient was a 30 year old woman who had three episodes of paroxysmal left lip movement 4 weeks after child delivery; MRI showed capillary telangiectasia in the right corticonuclear pathway. The third patient, a 36 year old man, had a transient right Bell's palsy; MRI disclosed two circumscribed areas consistent with capillary telangiectasia in the left corticospinal tract and medial longitudinal fasciculus.
\end{abstract}

Steroid receptors in the telangiectatic vessels walls might account for the recurrent and transient course seen in our two female patients.

Awareness of the MRI features of capillary telangiectasia may help in defining the real incidence, clinical correlation, and the risk of haemorrhagic complications of these vascular malformations. (F Neurol Neurosurg Psychiatry 2001;71:390-393)

Keywords: telangiectasia; vascular anomalies; brain stem; magnetic resonance imaging

Capillary telangiectasia consist of irregular clusters of dilated capillaries intermixed with normal brain parenchyma and are most often located in the pons. ${ }^{1}$ Based on their relatively common incidental discovery at necropsy in people without overt neurological manifestations, brain stem capillary telangiectasia were traditionally considered benign asymptomatic vascular anomalies. ${ }^{1-3}$ Due to the extremely slow flow, their demonstration in vivo is not possible with arteriography, but can be obtained with MRI. ${ }^{2}$ We report on three patients with symptomatic brain stem capillary telangiectasia unrelated to vascular rupture.

\section{Case reports}

CASE 1

A 28 year old woman presented with a left ear tinnitus which awoke her one night. The tinnitus, which she described as a roaring of a van, recurred during the next months with catamenial exacerbation. Neurological examination showed a mild weakness of the right arm and leg with diffusely increased deep tendon reflexes. Her hearing was normal. Routine blood laboratory tests, search for anti-DNA, anti-ANA, and anti-ENA antibodies, and results of coagulation studies were unremarkable. Somatosensory evoked potentials showed prolonged latencies and reduced amplitude of N20 and P39 waves on the right side. Brain stem auditory evoked potentials showed prolonged interwave I-V latency on the left. Brain MRI at $0.5 \mathrm{~T}$ showed an oval shaped mottled hyperintensity in proton density and T2 weighted images in the left paramedian region at the pontomesencephalic junction (fig 1). No other brain abnormalities were seen. The area of signal change enhanced after intravenous contrast administration (fig 1). She was examined 2 weeks later on a $1.5 \mathrm{~T}$ system using a gradient echo $\mathrm{T} 2{ }^{\star}$ which showed hypointensity in the brain stem lesion (fig 1). Tinnitus and neurological and MRI findings were unchanged 5 years later.

\section{CASE 2}

A 30 year old woman without relevant history complained of paroxysmal left lip movements, which suddenly appeared 4 weeks after the delivery of her first child. The involuntary movements spontaneously subsided 5 hours later but recurred twice in the next week. Neurological examination and EEG were normal. Routine blood laboratory tests, search for anti-DNA, anti-ANA, and anti-ENA antibodies, and results of coagulation studies were unremarkable. Motor, somatosensory, and brain stem auditory evoked potentials were normal. MRI at 1.5 Tesla showed a focal mottled area of hyperintensity in T2 weighted 

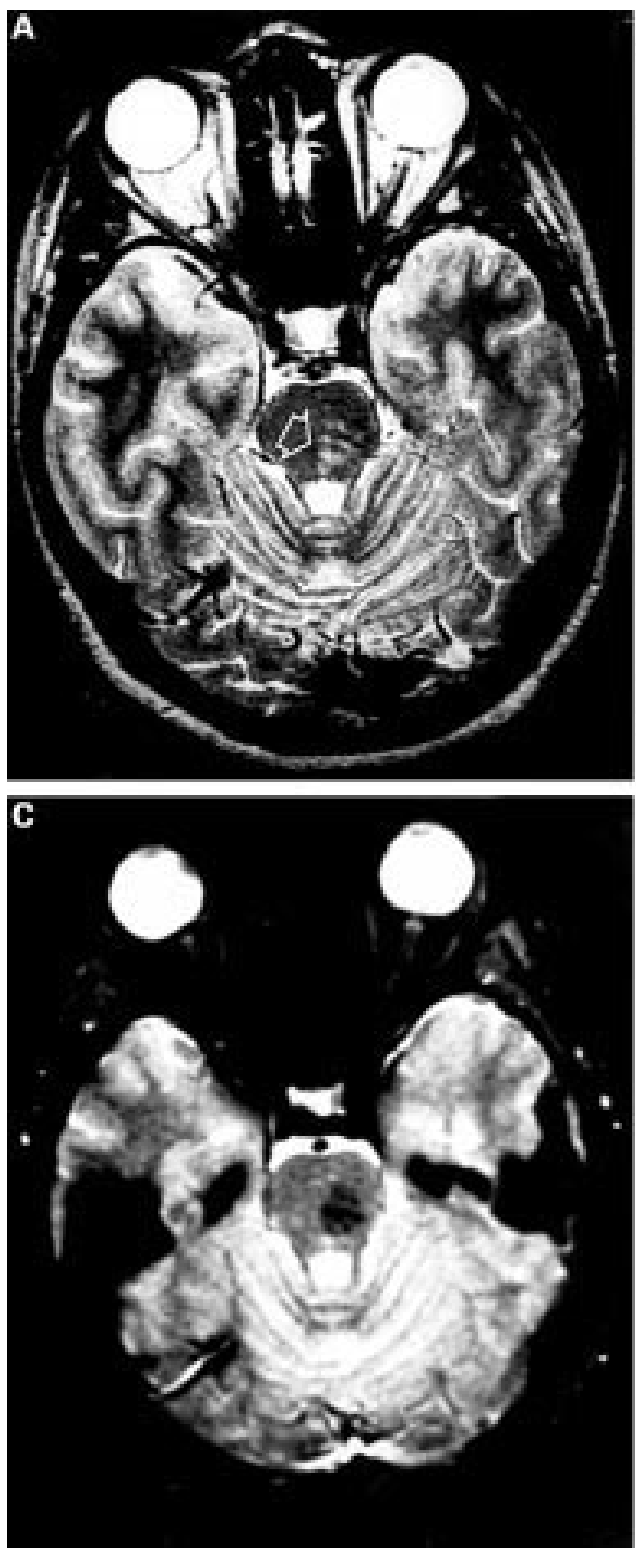

images in the lower right side of the pons which enhanced after intravenous contrast administration. No other brain abnormalities were found. Six months later symptoms had not recurred, neurological examination was negative, and MRI findings were unchanged; gradient echo $\mathrm{T} 2{ }^{\star}$ weighted sequence at $1.5 \mathrm{~T}$ showed hypointensity of the pontine lesion.

CASE 3

A 36 year old man presented with right Bell's palsy. Neurological examination showed complete right facial palsy. Routine blood and CSF laboratory analysis were unremarkable. He was treated with prednisone and the facial nerve palsy almost recovered in a few days. Cranial MRI at $0.5 \mathrm{~T}$ showed two small dots hyperintense in proton density weighted images which enhanced after intravenous contrast administration in the left paramedian central portion of the basis pontis. No other brain abnormalities were found. Brain MRI at $1.5 \mathrm{~T} 1$ month later showed hypointensity of the dots in gradient echo $\mathrm{T} 2{ }^{\star}$ weighted sequences.

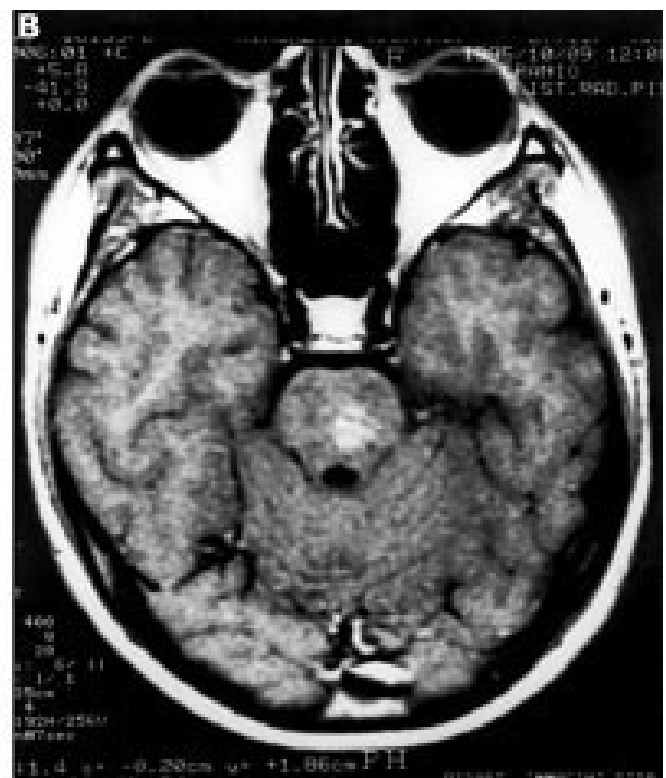

Figure 1 Patient 1.Unruptured capillary telangectasia of the brain stem. (A) Axial T2 weighted (TR $4000 \mathrm{~ms}$, TE $85 \mathrm{~ms}$, echo train length 12) turbo spin echo image demonstrates mottled hyperintensity (arrow) in the left paramedian pontomesencephalic junction. (B) The area shows contrast enhancement in an axial T1 weighted (TR $400 \mathrm{~ms}$, TE $20 \mathrm{~ms}$ ) spin echo image after intravenous administration of Gd-DTPA. (C) Low signal intensity is seen in the same area in an axial T2* weighted (TR 600 $m s$, TE $25 \mathrm{~ms}$, flip angle 20) gradient echo image.

\section{Discussion}

Capillary telangiectasia have characteristic MRI features which enable their in vivo detection and differentiation from other brain stem diseases including multiple sclerosis, infarction, and neoplasm. ${ }^{2}$ These features reflect the extremely slow flow in the vessels and the normality of the intermixed brain parenchyma. Accordingly, the vessels appear as isointense or hypointense areas compared with the normal brain parenchyma in $\mathrm{T} 1$ weighted unenhanced spin echo images and as isointense or hyperintense areas in proton density and T2 weighted spin echo and fast spin echo images. Above all, they exhibit hypointensity in $\mathrm{T} 2^{\star}$ weighted gradient echo images, especially if obtained on high field $(\geqslant 1.0 \mathrm{~T})$ MRI systems. This appearance reflects the higher sensitivity of gradient echo sequences to the high deoxyhaemoglobin content of the stagnant blood in the abnormal vessels, which determines a shortening of $\mathrm{T} 2$ relaxation time of blood and a decrease in signal. ${ }^{2}{ }^{3}$ Typically, the vessels enhance after contrast administration creating a mesh of enhanced structures on a 


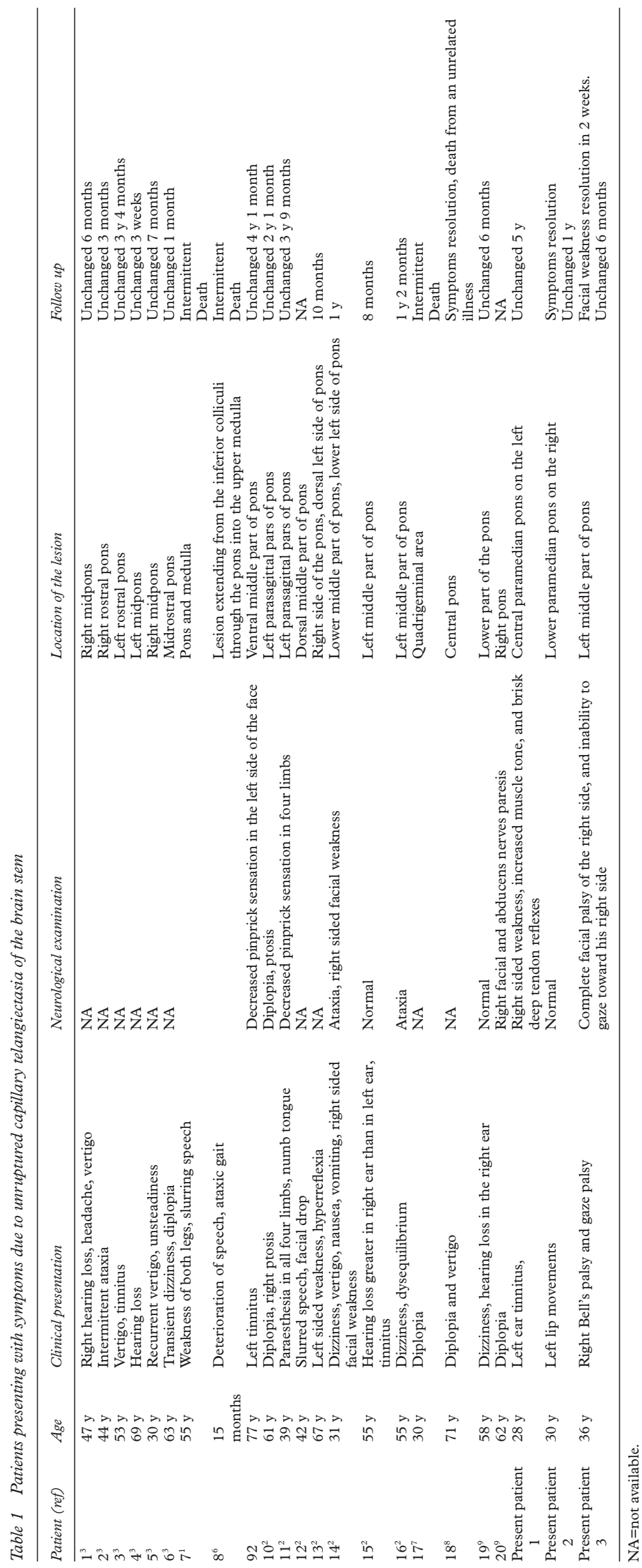

background of unenhanced brain parenchyma. The stability of the MRI features over time is a relevant clue in distinguishing capillary telangiectasia from haemorrhage, in which complex signal intensity patterns evolve in an orderly fashion. ${ }^{4}$ Occasionally, capillary telangiectasia are associated with venous anomalies. ${ }^{2}$

The frequency of brain stem capillary telangiectasia is unknown. In 1968 McCormick et $a \bar{l}$ reported 27 pathologically verified cases of pontine telangiectasia in a series of 164 vascular malformations of the posterior cranial fossa. More recently Barr et $a l^{\beta}$ and Lee et $a l^{2}$ described the clinical and MRI features of 12 and 15 cases of pontine telangiectasia. Over a period of 5 years we found, in addition to the three symptomatic patients reported herein, two more cases of brain stem capillary telangiectasia demonstrated by MRI in which no clinical counterpart was found. One patient was examined for a pituitary amenorrhoea and the other for headache.

In a review of the English literature we found 20 cases of unruptured brain stem capillary telangiectasia presenting with transient or permanent symptoms compatible with lesion location demonstrated by pathology, CT, or MRI..$^{1-3}{ }^{6-9}$ Location and presenting symptoms in these 20 cases along with our three cases are summarised in table 1 . The age of presentation ranged from 15 months to 71 years, but was predominantly in the third and fourth decade. Symptoms included vertigo or diplopia in five of $20(25 \%)$ patients, hearing loss, dizziness, focal weakness, or ataxia in four $(20 \%)$, tinnitus, or speech disturbances in three (15\%), hyperreflexia, monocular ptosis, and paraesthesia in one instance each. ${ }^{1-3}$ 6-9

In our three patients the lesion location was consistent with clinical features. In case 1 the malformation involved the acoustic pathway (lateral lemniscus), possibly explaining the tinnitus and the prolonged interwave I-V latency of the brain stem auditory evoked potentials, on the same side. The involvement of the right corticonuclear fasciculus might explain the abnormal lip movement in our case 2. In case 3 the involvement of the left corticonuclear tract explains the Bell's palsy.

In our findings and in previously reported cases, ${ }^{1-3}$ 6-9 symptoms related to brain stem capillary telangiectasia had a transient or intermittent course resembling transient ischaemic attacks or inflammatory diseases.

The pathophysiology underlying transient or intermittent symptoms in patients with capillary telangiectasia is not established. It is noteworthy that in two of our patients, symptoms developed or were exacerbated during the menstrual period or after pregnancy. Some vascular malformations, such as orbital angioma, present steroid receptors in both muscular and endothelial cells. ${ }^{10}{ }^{11}$ We submit that stimulation of steroids receptors expressed by endothelial cells in telangiectasia could be the triggering event of neurological symptoms through a vasomotor or a haemodynamic mechanism. 
A difficult problem related to frequency and natural history of brain stem capillary telangiectasia concerns the possibility that these vascular anomalies present dramatically with vascular rupture without any possibility of documenting the native vascular malformation that is cancelled out by the haemorrhage. ${ }^{12}$ The frequency of haemorrhagic complication of capillary telangiectasia is unknown. Although instances of intraparenchymal or subarachnoid haemorrhages due to ruptured capillary telangectasias are reported in the literature, ${ }^{12-15}$ haemorrhagic complications did not occur, neither in 27 patients of MRI documented brain stem capillary telangiectasia followed up for a period ranging from 1 month to 4 years, ${ }^{23}$ nor in our patients. Furthermore, none of the 27 cases of pontine capillary telangiectasia reported by McCormick et $a \bar{P}$ were associated with significant haemorrhage.

Awareness of the MRI features of capillary telangiectasia may help in defining the real incidence and clinical correlates of this vascular malformation. In addition, longitudinal studies might help to assess the risk of vascular rupture.

1 Farrel DF, Forno LS. Symptomatic capillary telangiectasis of the brainstem without hemorrhage. Report of an unusual case. Neurology 1970;20:341-6.
2 Lee RR, Becher MW, Benson ML, et al. Brain capillary elangiectasia: MR imaging appearance and clinicohistopathologic findings. Radiology 1997;205:797-805.

3 Barr RM, Dillon WP, Wilson CB. Slow-flow vascular malformations of the pons: capillary telangiectasias? AfNR Am $\mathcal{F}$ Neuroradiol 1996;17:71-8.

4 Alkemper T. Hemorrhage. In: Reimer P, Parizel PM, Stichnoth FA. Clinical MR imaging. A pratical approach. Berlin: Springer, 1999:49-60.

5 McCormick WF, Hardman JM, Boulter TR. Vascular malformations ("angiomas") of the brain, with special reference to those occurring in the posterior fossa. 7 Neurosurg 1968;28:241-51.

6 Huddle DC, Chaloupka JC, Sehgal V. Clinically aggressive diffuse capillary telangiectasia of the brain stem: a clinical radiologic-pathologic case study. AfNR Am 7 Neuroradiol radiologic-pathologi

7 Stahl S, Johnson KP, Malamud N. The clinical and pathological spectrum of brain-stem vascular malformation. Long term course simulates multiple sclerosis. Arch Neurol 1980;37:25-9.

8 Kuzma B, Goodman JM, Britt P. Capillary telangiectasia of the pons. Surg Neurol 1997;48:93-4.

9 Kuker W, Nacimiento W, Block F, Thron A. Presumed capillary telangectasia of the pons: MRI and follow-up. Eur Radiol 2000;10:945-50.

10 Ditommaso L, Scarpellini F, Salvi F, et al. Progesterone receptor expression in orbital cavernous hemangiomas. receptor expression in orbital
Virchows Arch 2000;436:284-8

11 Gazzaz M, Sichez JP, Capelle L, et al. Saignements itératifs d'un angiome caverneux sous traitment hormonal. A propos d'un cas. Neurochirurgie 1999;45:413-16.

12 Howard RS. Brainstem haematoma due to presumed cryptic telangiectasia. I Neurol Neurosurg Psychiatry 1986;49 $1241-5$.

3 Teilmann K. Hemangiomas of the pons. Arch Neurol Psych 1953;69:208-23

14 El-Gohary EM, Tomita T, Gutierrez FA, et al. Angiographically occult vascular malformations in childhood. Neurosurgery 1987;20:759-66.

15 Wijdicks EFM, Schievinick WI. Perimesencephalic nonaneurysmal subarachnoid hemorrhage: first hint of a cause? Neurology 1997;49:634-6. 